\author{
Marquette University \\ e-Publications@Marquette
}

\title{
An Analytical Model for Transient Deformation of Viscoelastically Coated Beams: Applications to Static-mode Microcantilever Chemical Sensors
}

\author{
Stephen M. Heinrich \\ Marquette University, stephen.heinrich@marquette.edu \\ Michael J. Wenzel \\ Marquette University \\ Fabien Josse \\ Marquette University, fabien.josse@marquette.edu \\ Isabelle Dufour \\ Université de Bordeaux
}

Follow this and additional works at: https://epublications.marquette.edu/civengin_fac

Part of the Civil and Environmental Engineering Commons

\section{Recommended Citation}

Heinrich, Stephen M.; Wenzel, Michael J.; Josse, Fabien; and Dufour, Isabelle, "An Analytical Model for Transient Deformation of Viscoelastically Coated Beams: Applications to Static-mode Microcantilever Chemical Sensors" (2009). Civil and Environmental Engineering Faculty Research and Publications. 71. https://epublications.marquette.edu/civengin_fac/71 


\title{
An analytical model for transient deformation of viscoelastically coated beams: Applications to static-mode microcantilever chemical sensors
}

\author{
S. M. Heinrich, ${ }^{1, a)}$ M. J. Wenzel, ${ }^{2}$ F. Josse, ${ }^{2}$ and I. Dufour ${ }^{3}$ \\ ${ }^{1}$ Department of Civil and Environmental Engineering, Marquette University, P.O. Box 1881, Milwaukee, \\ Wisconsin 53201, USA \\ ${ }^{2}$ Department of Electrical and Computer Engineering, Marquette University, P.O. Box 1881, Milwaukee, \\ Wisconsin 53201, USA \\ ${ }^{3}$ Laboratoire IMS, Université de Bordeaux, 351 Cours de la Libération, 33405 Talence Cedex, France
}

(Received 27 August 2008; accepted 8 May 2009; published online 17 June 2009)

\begin{abstract}
The problem governing the transient deformation of an elastic cantilever beam with viscoelastic coating, subjected to a time-dependent coating eigenstrain, is mathematically formulated. An analytical solution for an exponential eigenstrain history, exact within the context of beam theory, is obtained in terms of the coating and base layer thicknesses, the elastic modulus of the base material, the initial coating modulus, the coating relaxation percentage $(0 \%-100 \%)$, and the time constants of the coating's relaxation process and its eigenstrain history. Approximate formulas, valid for thin coatings, are derived as special cases to provide insight into system behavior. Main results include (1) the time histories of the beam curvature and the coating stresses, (2) a criterion governing the response type (monotonic or "overshoot" response), and (3) simple expressions for the overshoot ratio, defined as the peak response scaled by the steady-state response, and the time at which the peak response occurs. Applications to polymer-coated microcantilever-based chemical sensors operating in the static mode are discussed. (C) 2009 American Institute of Physics.
\end{abstract}

[DOI: $10.1063 / 1.3148291]$

\section{INTRODUCTION}

\section{A. Background}

In recent years the development of microcantilever (MC)-based chemical and biochemical sensors has created another important application area for theoretical models for understanding the deformation of coated beams. These sensors may be operated in a dynamic (resonant) or static mode. ${ }^{1,2}$ In the case of dynamic mode operation, the change in mass associated with the sorption of analyte into the selective beam coating causes a shift in resonant frequency, which may be correlated to the ambient concentration of the target substance. For the static-mode case, the sorption of analyte causes a quasistatic curvature that is often induced by the tendency of the coating to expand upon analyte sorption, not unlike the behavior of a bimetallic strip subjected to temperature change. In this mode of operation, the objective is to correlate the quasistatic MC sensor response (deflection) to the ambient analyte concentration; this, of course, requires a sufficiently accurate model of the coated-beam deformation.

When the coating material may be modeled as elastic (the case for most metals), the modeling of beam deformation (dynamic or static) is relatively straightforward. For the dynamic mode, the equivalent flexural rigidity of the elastic composite may be calculated using the concept of a transformed section, ${ }^{3}$ and this property may then be utilized in conjunction with classical solution methods for elastic canti-

\footnotetext{
${ }^{\text {a) }}$ Author to whom correspondence should be addressed. Electronic mail: stephen.heinrich@marquette.edu. Tel.: (414) 288-5466. FAX: (414) 2887521.
}

lever beam vibrations. ${ }^{4}$ Complications associated with elastic beam vibrations in a fluid medium have also been taken into consideration. ${ }^{5}$ For static-mode operation of MC chemical sensors, classical results such as Stoney's equation ${ }^{6}$ and Timoshenko's bimetallic strip solution, ${ }^{7}$ as well as several extensions of these results, ${ }^{8-18}$ may be applied; however, most of these solutions are based on the assumption that the coating is elastic.

In many cases of practical interest the coating material utilized in MC-based chemical sensors is a polymer, for which an elastic material model might not be sufficient due to the energy dissipation that occurs during polymer deformation. To account for these energy losses, polymers are often modeled as viscoelastic. ${ }^{19,20}$ The incorporation of coating viscoelasticity into dynamic-mode models of MC sensors is relatively simple. Once the effective complex flexural rigidity of the composite section is determined by one of several existing methods, ${ }^{21-23}$ one may utilize an appropriate form of correspondence principle for steady-state harmonic vibrations $^{24}$ to convert a dynamic solution for an elastic beam to the corresponding viscoelastic solution. ${ }^{25}$ However, for the static mode the effects of coating viscoelasticity are not handled as easily.

To the authors' knowledge, analytical solutions analogous to the classical elastic bilayer beam solutions of Stoney and Timoshenko do not exist for the case in which one of the layers is viscoelastic, yet such a solution is precisely what is needed to account for the viscoelasticity of polymer coatings in static-mode MC sensors. Moreover, as illustrated in Fig. 1, experimental data for polymer-coated, static-mode MC sensors may exhibit either a monotonic transient response or one that "overshoots" the steady-state deflection. ${ }^{1,26,27}$ While the 


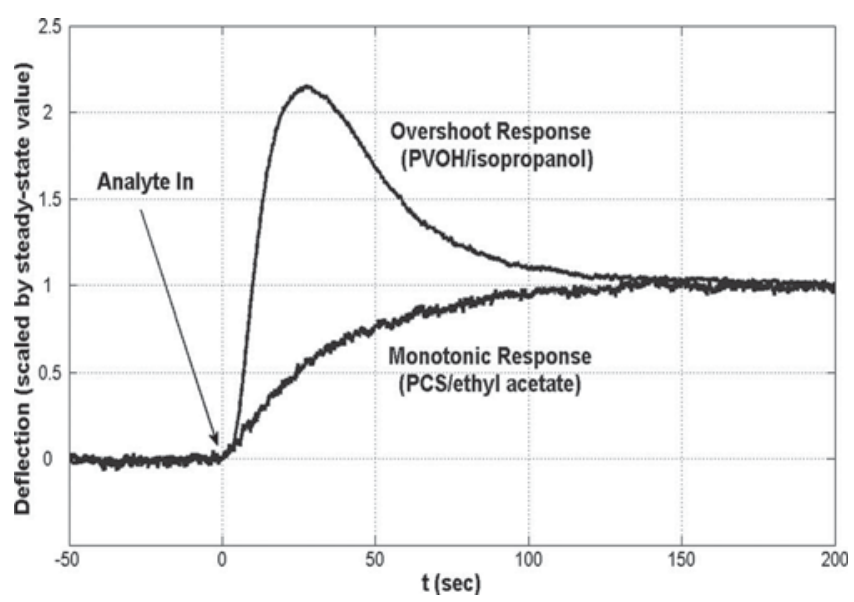

FIG. 1. Overshoot and monotonic responses for polymer-coated, staticmode MC-based chemical sensors. Data correspond to two different coating/ analyte pairs and are taken from Loui et al. (Ref. 27).

latter behavior is inconsistent with that predicted by elastic models during sorption, it may be explained by stress relaxation effects associated with coating viscoelasticity. This observation provides the motivation for the present study, whose goals are (1) to present a rigorous derivation of the initial-value problem (IVP) governing the deformation of an elastic cantilever beam with viscoelastic coating, subjected to an arbitrary coating "eigenstrain" history, and (2) to derive analytical solutions for the practical case in which the coating eigenstrain approaches its steady-state value exponentially in time, with a step function being included as a special limiting case.

In this paper the term eigenstrain is used to denote any stress-free straining that the coating would experience if it were not constrained by the base layer. While the physical source of the eigenstrain in static-mode, MC-based chemical sensors is analyte sorption, other sources of eigenstrain could include temperature change, hygroscopic swelling, phase transformation, misfit strains, etc. ${ }^{28,29}$ Therefore, the mathematical formulation and analytical solutions presented herein are of fundamental interest and need not be restricted to the modeling of MC-based chemical sensors.

\section{B. Related work}

In a recent paper by the authors ${ }^{30}$ the problem of a staticmode MC sensor with viscoelastic coating was formulated and solved using a numerical approach. Because the focus of that paper was to model the beam deformation caused by an arbitrary time history of ambient analyte concentration, including the modeling of sorption kinetics, coating viscoelasticity, and concentration-dependent coating plasticization, the formulation presented in the earlier paper was necessarily more general than that of the present work. As a result, a numerical solution of the governing equations was required.

Unlike the authors' recent work, the primary goal of the present paper is to derive analytical (closed-form) solutions to the problem of interest as such solutions (a) may provide insight into the specific roles that the various system parameters play in determining the response of viscoelastically coated beams and (b) may serve as valuable benchmark so-

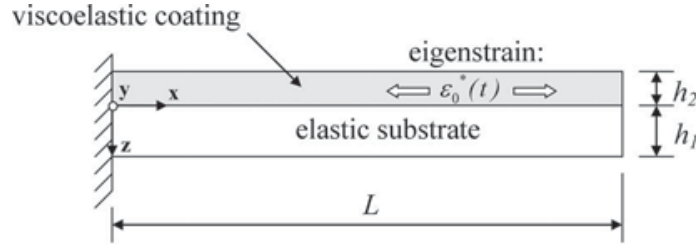

(a)

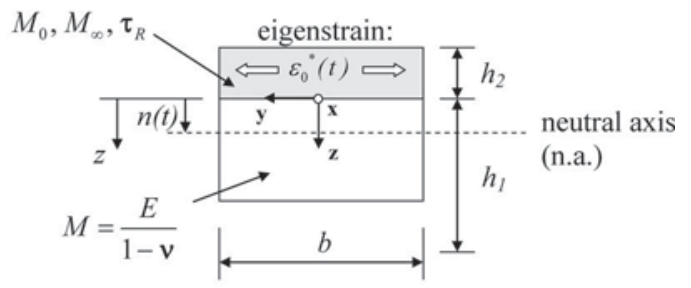

(b)

FIG. 2. Problem parameters for cantilever beam with viscoelastic coating under eigenstrain: (a) beam profile; (b) beam cross section.

lutions (e.g., for verifying numerical solution techniques). To this end, the problem of transient beam deformation will be formulated by assuming that the coating eigenstrain is specified a priori. (In the case of MC chemical sensor applications, this would require a theoretical understanding or experimental characterization of the sorption kinetics and the sorption-induced expansion associated with the analyte/ coating pair.) In addition, the governing IVP will be formulated in dimensionless form in order to minimize the number of independent parameters appearing in the analytical results and in the corresponding plots that are generated to display the system behavior.

\section{Specific objectives of present study}

The specific objectives of this study are (a) to mathematically formulate the problem of determining the response (i.e., stress, strain, curvature, and deflection histories) of a cantilever beam with viscoelastic coating when the coating is subjected to an arbitrary eigenstrain history, (b) to derive an "exact" analytical solution and an approximate "thin-coating solution" for the system response when the coating eigenstrain increases exponentially in time to its steady-state value, (c) to utilize the thin-coating solution to develop a simple "overshoot criterion" that may be used to predict if an overshoot (i.e., nonmonotonic) response will occur, and (d) to derive simple expressions to quantify the magnitude of the overshoot and the time at which the peak response occurs. Applications of the results that are relevant to MC-based chemical sensors will also be discussed.

\section{PROBLEM STATEMENT}

Consider an elastic cantilever beam of rectangular cross section coated with a viscoelastic layer (Fig. 2). The coating is subjected to a uniform eigenstrain history $\varepsilon_{0}^{*}(t)$ and the mechanical behavior of the coating is taken to be that of a three-parameter viscoelastic solid. The resulting deformation history of the beam [curvature $\kappa(t)$ or tip deflection $w(t)$ ] and the time-varying strain and stress profiles within the coated beam are to be determined. The problem parameters include 
$h_{1}$ and $h_{2}$, the thicknesses of the base layer and coating, respectively the beam length $L$ and width $b$, the base material's biaxial elastic modulus $M \equiv E /(1-\nu)$, where $E$ and $\nu$ are the (uniaxial) Young's modulus and Poisson's ratio of the base material, respectively, and the in-plane properties of the viscoelastic coating material, which include its instantaneous and asymptotic biaxial moduli $M_{0}$ and $M_{\infty}$, respectively, and the corresponding relaxation time constant $\tau_{R}$, all three of which will be explained subsequently. The only load on the system is the coating eigenstrain $\varepsilon_{0}^{*}(t)$. The time-dependence of the solution will arise due to the time-dependence of both the eigenstrain and the creep/relaxation behavior of the viscoelastic coating material.

Of particular interest in this study is the derivation of an overshoot criterion for determining what combinations of problem parameters will result in a beam response that exhibits an overshoot phenomenon. Moreover, in order to quantify the overshoot, expressions will be derived for (a) the "critical time" $t_{\text {cr }}$ at which the peak curvature (or deflection) occurs and (b) a response parameter referred to as the "overshoot ratio (OSR)," defined as

$$
\mathrm{OSR} \equiv \frac{\kappa_{\max }}{\kappa(\infty)}
$$

i.e., the ratio of the maximum beam curvature to the steadystate curvature. Definition (1) implies that a monotonically increasing curvature (no overshoot) would result in $\mathrm{OSR}=1$, while for a response exhibiting overshoot, the value of the OSR would exceed unity and its value would characterize the magnitude of the overshoot.

\section{FORMULATION OF INITIAL-VALUE PROBLEM}

\section{A. Assumptions}

The mathematical formulation of the title problem will be based on the following assumptions: (1) Planar cross sections remain planar and normal to the deformed beam axis (Bernoulli-Euler assumption of elementary beam theory) and beam rotations (slopes) are small; ${ }^{3}(2)$ the base material is elastic, while the in-plane behavior of the coating material is assumed to be that of a three-parameter viscoelastic solid; ${ }^{19}$ (3) the coating eigenstrain is uniform, has a specified time-history, and is isotropic within the $x-y$ plane (Fig. 2); (4) perfect bond exists between coating and base layer [a result that follows from assumption (1)]; (5) the beam is initially straight and in a state of zero stress and strain prior to the introduction of the coating eigenstrain; (6) edge effects due to the clamped support at $x=0$ and the traction-free surfaces at $x=L$ and $y= \pm b / 2$ are not considered (Fig. 2). (As a result, the stress distributions derived herein are not expected to include localized effects near the free end of the cantilever, but these effects are assumed to have a negligible effect on the beam curvature and deflection for sufficiently long beams.); (7) as the present model will be based on the kinematic assumptions of beam theory, the localized stresses associated with the interface between the coating and the base material will not be considered, and their effects on the stresses away from the interface and on the overall beam deformation are assumed to be negligible; (8) inertial effects are ignored.

\section{B. Derivation of governing equations}

For completeness and clarity, a summary of relevant portions of the authors' previous derivation ${ }^{30}$ of the governing equations will be given in the present section. As a direct result of the Bernoulli-Euler assumption, the total longitudinal strain on the beam cross section, $\varepsilon_{\text {tot }}(z, t)$, will vary linearly along the beam depth and be related to the beam curvature $\kappa(t)$ as follows:

$$
\varepsilon_{\mathrm{tot}}(z, t)=-\kappa(t)[z-n(t)],
$$

where $z$ is measured downward from the interface and $n(t)$ denotes the value of $z$ corresponding to the neutral-axis position at time $t$ (Fig. 2). Equation (2) is based on the convention that positive curvature is concave downward (downward cantilever deflection). The neutral axis is defined to be the locus of points on the cross section for which $\varepsilon_{\text {tot }}$ vanishes. This total strain may be decomposed into the (stress-free) eigenstrain $\varepsilon^{*}(z, t)$ and the stress-related strain, denoted by $\varepsilon(z, t)$ :

$$
\varepsilon_{\mathrm{tot}}(z, t)=\varepsilon(z, t)+\varepsilon^{*}(z, t) .
$$

Employing the assumptions that only the coating is subjected to an eigenstrain and that this eigenstrain is uniform, Eqs. (2) and (3) result in

$$
\varepsilon(z, t)= \begin{cases}-\kappa(t)[z-n(t)]-\varepsilon_{0}^{*}(t), & -h_{2} \leqslant z<0, \\ -\kappa(t)[z-n(t)], & 0<z \leqslant h_{1},\end{cases}
$$

where $\varepsilon_{0}^{*}(t)$ is the specified eigenstrain. It can be shown that the strain distribution of Eqs. (4a) and (4b) implies a bilinear stress distribution of the form ${ }^{30}$

$$
\sigma(z, t)= \begin{cases}\left(1+\frac{z}{h_{2}}\right) \sigma_{c}^{\mathrm{bot}}(t)-\frac{z}{h_{2}} \sigma_{c}^{\mathrm{top}}(t), & -h_{2} \leqslant z<0, \\ -M \kappa(t)[z-n(t)], & 0<z \leqslant h_{1},\end{cases}
$$

where $\sigma_{c}^{\text {bot }}(t)$ and $\sigma_{c}^{\text {top }}(t)$ are the stresses at the bottom and top of the coating. Note that the biaxial modulus, $M$ $\equiv E /(1-\nu)$, has been used in Eq. (5b) to reflect the stiffening effect associated with plate behavior, which results from eigenstrain components along both the $x$ - and $y$-directions. ${ }^{8,28}$ (See assumption 3.)

Because the beam is not subjected to any external mechanical loads, the resultant axial force and bending moment on the cross section must be zero. Imposing these conditions on Eqs. (5a) and (5b) results in the following expressions relating the neutral-axis position and the beam curvature to $\sigma_{c}^{\mathrm{bot}}(t)$ and $\sigma_{c}^{\mathrm{top}}(t)$ :

$$
\begin{aligned}
& n(t)=h_{1} \frac{\left(2 h_{1}+h_{2}\right) \sigma_{c}^{\mathrm{bot}}(t)+\left(2 h_{1}+2 h_{2}\right) \sigma_{c}^{\mathrm{top}}(t)}{\left(3 h_{1}+2 h_{2}\right) \sigma_{c}^{\mathrm{bot}}(t)+\left(3 h_{1}+4 h_{2}\right) \sigma_{c}^{\mathrm{top}}(t)}, \\
& \kappa(t)=-\frac{h_{2}}{M h_{1}^{3}}\left[\left(3 h_{1}+2 h_{2}\right) \sigma_{c}^{\mathrm{bot}}(t)+\left(3 h_{1}+4 h_{2}\right) \sigma_{c}^{\mathrm{top}}(t)\right] .
\end{aligned}
$$




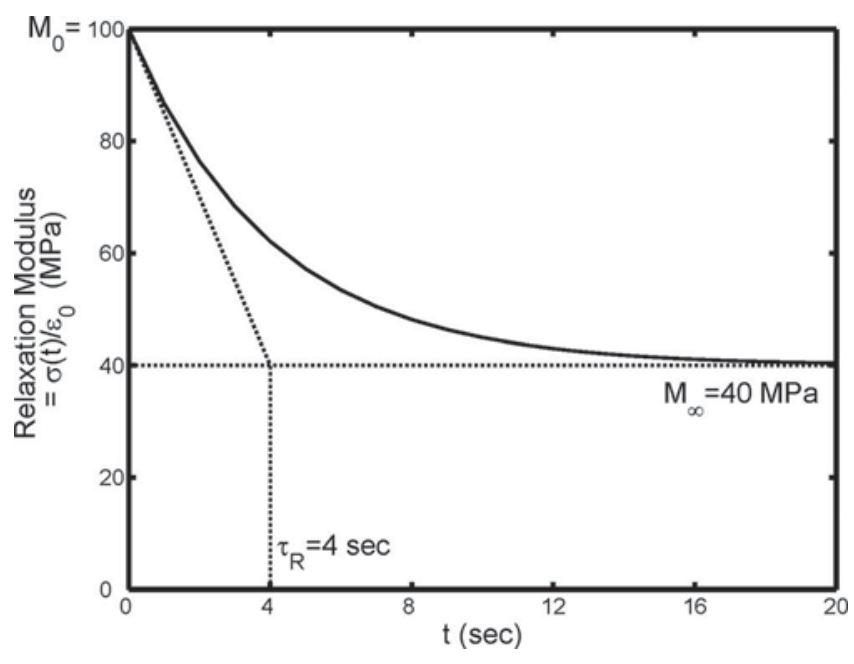

FIG. 3. Plot of the biaxial relaxation modulus of a hypothetical threeparameter viscoelastic solid. Biaxial stress $\sigma(t)$ corresponds to an imposed step-function biaxial strain of magnitude $\varepsilon_{0}$.

Next, a particular constitutive law will be introduced so that the coating stresses and strains may be related. Using the three-parameter solid model for the coating material, the stress and stress-related strain in the coating are related by ${ }^{19}$

$$
\sigma+\tau_{R} \frac{d \sigma}{d t}=M_{\infty} \varepsilon+\tau_{R} M_{0} \frac{d \varepsilon}{d t},
$$

where $M_{0}$ and $M_{\infty}$ are the instantaneous and asymptotic biaxial moduli of the coating material and $\tau_{R}$ is the relaxation time constant of the coating material associated with an isotropic, biaxial loading. The physical meanings of these coating parameters are indicated in Fig. 3. Note that parameter $\tau_{R}$ provides a measure of how quickly the coating stress relaxes (under constant strain), with larger $\tau_{R}$ corresponding to slower relaxation.

The strain in the coating may be expressed in terms of the coating stresses by substituting Eqs. (6) and (7) into Eq. (4a). When the resulting strain expression is placed into Eq. (8), the latter may be evaluated at the top and bottom of the coating. This yields the equations governing $\sigma_{c}^{\text {bot }}(t)$ and $\sigma_{c}^{\text {top }}(t)$ for a specified coating eigenstrain $\varepsilon_{0}^{*}(t)$,

$$
\begin{aligned}
& {\left[\begin{array}{cc}
{\left[1+\bar{M}_{0} f_{1}(\beta)\right]} & \bar{M}_{0} f_{2}(\beta) \\
\bar{M}_{0} f_{3}(\beta) & {\left[1+\bar{M}_{0} f_{4}(\beta)\right]}
\end{array}\right]\left\{\begin{array}{c}
\dot{\bar{\sigma}}_{c}^{\mathrm{bot}}(\bar{t}) \\
\dot{\bar{\sigma}}_{c}^{\mathrm{top}}(\bar{t})
\end{array}\right\}} \\
& +\left[\begin{array}{cc}
{\left[1+\bar{M}_{\infty} f_{1}(\beta)\right]} & \bar{M}_{\infty} f_{2}(\beta) \\
\bar{M}_{\infty} f_{3}(\beta) & {\left[1+\bar{M}_{\infty} f_{4}(\beta)\right]}
\end{array}\right]\left\{\begin{array}{c}
\bar{\sigma}_{c}^{\mathrm{bot}}(\bar{t}) \\
\bar{\sigma}_{c}^{\mathrm{top}}(\bar{t})
\end{array}\right\} \\
& =-\left[\begin{array}{cc}
\bar{M}_{\infty} & \bar{M}_{0} \\
\bar{M}_{\infty} & \bar{M}_{0}
\end{array}\right]\left\{\begin{array}{c}
\varepsilon_{0}^{*}(\bar{t}) \\
\dot{\varepsilon}_{0}^{*}(\bar{t})
\end{array}\right\} \text {, }
\end{aligned}
$$

where

$$
\begin{aligned}
& f_{1}(\beta) \equiv \beta^{2}+2 \beta \\
& f_{2}(\beta) \equiv 2 \beta^{2}+2 \beta \\
& f_{3}(\beta) \equiv 2 \beta^{3}+4 \beta^{2}+2 \beta
\end{aligned}
$$

$$
f_{4}(\beta) \equiv 4 \beta^{3}+5 \beta^{2}+2 \beta
$$

The following normalized quantities have been introduced to render the governing equations dimensionless:

$$
\begin{aligned}
& \bar{\sigma} \equiv \frac{\sigma}{M}, \\
& \bar{t} \equiv \frac{t}{\tau_{R}}, \\
& \beta \equiv \frac{h_{2}}{h_{1}}, \\
& \bar{M}_{0} \equiv \frac{M_{0}}{M}, \\
& \bar{M}_{\infty} \equiv \frac{M_{\infty}}{M} .
\end{aligned}
$$

[The "dot" notation in Eq. (9) denotes differentiation with respect to $\bar{t}$.] The assumption that the system is initially stress-free leads to the following initial conditions:

$$
\bar{\sigma}_{c}^{\mathrm{bot}}(0)=\bar{\sigma}_{c}^{\mathrm{top}}(0)=0 .
$$

Equations (9) and (12) represent an IVP that may be solved for a specified input $\varepsilon_{0}^{*}(\bar{t})$. Once the coating stress histories, $\bar{\sigma}_{c}^{\mathrm{bot}}(\bar{t})$ and $\bar{\sigma}_{c}^{\mathrm{top}}(\bar{t})$, are obtained, the neutral-axis position and beam curvature are given by the normalized forms of Eqs. (6) and (7),

$$
\begin{aligned}
& \bar{n}(\bar{t})=\frac{(2+\beta) \bar{\sigma}_{c}^{\mathrm{bot}}(\bar{t})+(2+2 \beta) \bar{\sigma}_{c}^{\mathrm{top}}(\bar{t})}{(3+2 \beta) \bar{\sigma}_{c}^{\mathrm{bot}}(\bar{t})+(3+4 \beta) \bar{\sigma}_{c}^{\mathrm{top}}(\bar{t})}, \\
& \bar{\kappa}(\bar{t})=-\left[\left(2 \beta^{2}+3 \beta\right) \bar{\sigma}_{c}^{\mathrm{bot}}(\bar{t})+\left(4 \beta^{2}+3 \beta\right) \bar{\sigma}_{c}^{\mathrm{top}}(\bar{t})\right],
\end{aligned}
$$

where $\bar{n} \equiv n / h_{1}$ and $\bar{\kappa} \equiv h_{1} \kappa$. If desired, the (normalized) stress profile throughout the cross section may be obtained by substituting $\bar{\sigma}_{c}^{\mathrm{bot}}(\bar{t}), \bar{\sigma}_{c}^{\mathrm{top}}(\bar{t}), \bar{n}(\bar{t})$, and $\bar{\kappa}(\bar{t})$ into the normalized form of Eqs. (5a) and (5b). Similarly, the strain profile is given by placing $\bar{n}(\bar{t})$ and $\bar{\kappa}(\bar{t})$ into the normalized form of Eqs. (4a) and (4b) for $\varepsilon$, or into Eq. (2) for $\varepsilon_{\text {tot }}$.

The dimensionless formulation is convenient as it clearly illustrates that, for a given eigenstrain history, $\varepsilon_{0}^{*}(\bar{t})$, the history of any normalized response quantity (stress, strain, curvature) depends on only three normalized parameters: the thickness ratio $\beta$ and the normalized biaxial coating moduli, $\bar{M}_{0}$ and $\bar{M}_{\infty}$.

By virtue of the assumptions that the deformation is small and the eigenstrain is uniform, the curvature may be integrated twice with respect to $x$ to yield the deflection at the free end,

$$
w(t)=\frac{1}{2} \kappa(t) L^{2}
$$

This may be expressed in dimensionless form as

$$
\bar{w}(\bar{t})=\bar{\kappa}(\vec{t}),
$$

in which $\bar{w} \equiv 2 h_{1} w / L^{2}$. Thus, the dimensionless curvature is the same as the normalized tip deflection. 


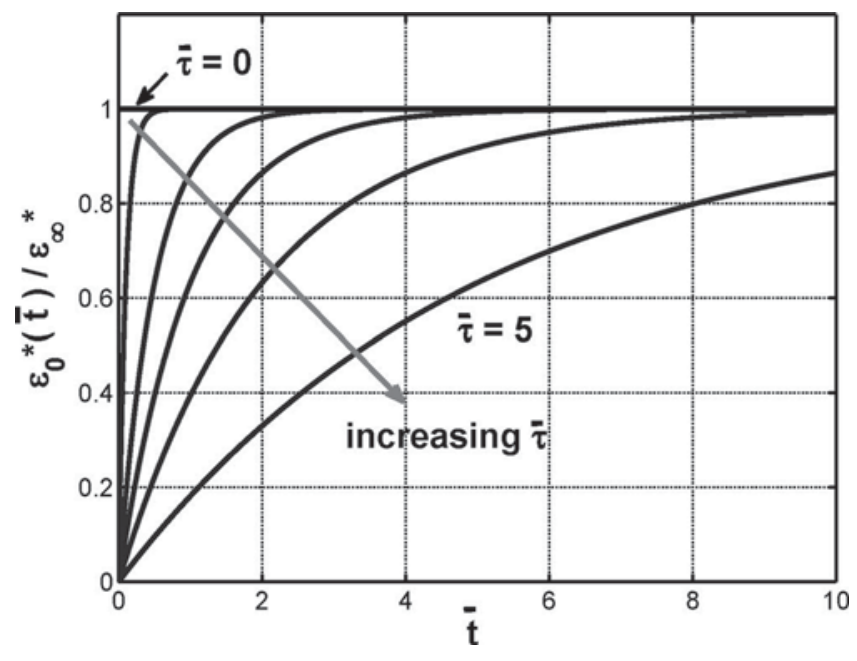

FIG. 4. Exponential eigenstrain histories (normalized form): $\varepsilon_{0}^{*}(\bar{t})=\varepsilon_{\infty}^{*}\left(1-e^{-\bar{t} / \bar{\tau}}\right)$. Increment in $\bar{\tau}$ is 1 .

\section{ANALYTICAL SOLUTIONS}

\section{A. Assumed loading: Exponential coating eigenstrain history}

In order to solve the IVP a specific form of eigenstrain must be assumed. A plausible form is

$$
\varepsilon_{0}^{*}(t)=\varepsilon_{\infty}^{*}\left(1-e^{-t / \tau_{\varepsilon}}\right),
$$

where $\varepsilon_{\infty}^{*}$ is the steady-state value of coating eigenstrain and $\tau_{\varepsilon}$ is the eigenstrain time constant that characterizes the rate at which the steady-state eigenstrain is approached. Note that, if the coated cantilever is designed as a chemical sensor such that the eigenstrain source is an absorbed target substance, then the form of Eq. (16a) is consistent with the assumptions that (a) the ambient analyte concentration is a step function in time and (b) the absorption rate is proportional to the difference between the ambient and coating concentrations. $^{30}$ Expressing the eigenstrain in terms of $\bar{t}$ yields

$$
\varepsilon_{0}^{*}(\bar{t})=\varepsilon_{\infty}^{*}\left(1-e^{-\bar{t} / \bar{\tau}}\right),
$$

where

$$
\bar{\tau} \equiv \frac{\tau_{\varepsilon}}{\tau_{R}}
$$

is the "relative time constant" of the eigenstrain history with respect to the coating relaxation. Plots of Eq. (16b) for vari- ous values of $\bar{\tau}$ are shown in Fig. 4. A small (large) value of $\bar{\tau}$ corresponds to an eigenstrain history that occurs relatively quickly (slowly) compared with the coating's relaxation process. The limiting case of $\bar{\tau} \rightarrow 0$, for which the eigenstrain history reduces to a step function, shall be referred to as the "rapid-eigenstrain" case.

\section{B. Exact solution for arbitrary coating thickness}

An exact solution to the IVP may be obtained by classical means when the input eigenstrain is given by Eq. (16b). One may confirm by direct substitution into Eqs. (9) and (12) that the exact solution may be expressed as

$$
\bar{\sigma}_{c}^{\mathrm{bot}}(\bar{t})=-\varepsilon_{\infty}^{*}\left[c_{0}\left(\bar{M}_{\infty}-\bar{\zeta} c_{1} c_{2} e^{-\bar{t} / \bar{\tau}}\right)+C_{1} e^{-\lambda_{1} \bar{t}}+C_{2} e^{-\lambda_{2} \bar{t}}\right],
$$

$$
\begin{aligned}
\bar{\sigma}_{c}^{\mathrm{top}}(\bar{t})= & -\varepsilon_{\infty}^{*}\left[c_{0} c_{3}\left(\bar{M}_{\infty}-\bar{\zeta} c_{1} c_{4} e^{-\bar{t} / \bar{\tau}}\right)+\varphi_{1} C_{1} e^{-\lambda_{1} \bar{t}}\right. \\
& \left.+\varphi_{2} C_{2} e^{-\lambda_{2} \bar{t}}\right], \\
\bar{\kappa}(\bar{t})=- & {\left[\left(3 \beta+2 \beta^{2}\right) \bar{\sigma}_{c}^{\mathrm{bot}}(\bar{t})+\left(3 \beta+4 \beta^{2}\right) \bar{\sigma}_{c}^{\mathrm{top}}(\bar{t})\right], }
\end{aligned}
$$

where

$$
\begin{aligned}
\bar{\zeta} & \equiv\left[\frac{1-\left(\frac{\bar{M}_{\infty}}{\bar{M}_{0}}\right) \bar{\tau}}{1-\bar{\tau}}\right] \bar{M}_{0}, \\
c_{0} & \equiv \frac{1+\left(3 \beta^{2}+4 \beta^{3}\right) \bar{M}_{\infty}}{1+\left(4 \beta+6 \beta^{2}+4 \beta^{3}\right) \bar{M}_{\infty}+\beta^{4} \bar{M}_{\infty}^{2}},
\end{aligned}
$$$$
c_{1} \equiv \frac{1+\left(4 \beta+6 \beta^{2}+4 \beta^{3}\right) \bar{M}_{\infty}+\beta^{4} \bar{M}_{\infty}^{2}}{1+\left(4 \beta+6 \beta^{2}+4 \beta^{3}\right) \bar{\zeta}+\beta^{4} \bar{\zeta}^{2}},
$$$$
c_{2} \equiv \frac{1+\left(3 \beta^{2}+4 \beta^{3}\right) \bar{\zeta}}{1+\left(3 \beta^{2}+4 \beta^{3}\right) \bar{M}_{\infty}},
$$

$$
c_{3} \equiv \frac{1-\left(3 \beta^{2}+2 \beta^{3}\right) \bar{M}_{\infty}}{1+\left(3 \beta^{2}+4 \beta^{3}\right) \bar{M}_{\infty}},
$$

$$
c_{4} \equiv \frac{1-\left(3 \beta^{2}+2 \beta^{3}\right) \bar{\zeta}}{1-\left(3 \beta^{2}+2 \beta^{3}\right) \bar{M}_{\infty}},
$$

$$
\begin{aligned}
& \lambda_{1,2} \equiv \frac{1+\left(2 \beta+3 \beta^{2}+2 \beta^{3}\right)\left(\bar{M}_{0}+\bar{M}_{\infty}\right)+\bar{M}_{0} \bar{M}_{\infty} \beta^{4} \mp 2 \beta(1+\beta) \sqrt{1+\beta+\beta^{2}}\left(\bar{M}_{0}-\bar{M}_{\infty}\right)}{1+\left(4 \beta+6 \beta^{2}+4 \beta^{3}\right) \bar{M}_{0}+\beta^{4} \bar{M}_{0}^{2}} \quad\left(0 \leqslant \lambda_{1} \leqslant \lambda_{2}\right), \\
& \varphi_{i} \equiv \frac{\left[1+\bar{M}_{0}\left(2 \beta+\beta^{2}\right)\right] \lambda_{i}-\left[1+\bar{M}_{\infty}\left(2 \beta+\beta^{2}\right)\right]}{\left(2 \beta+2 \beta^{2}\right)\left(\bar{M}_{\infty}-\bar{M}_{0} \lambda_{i}\right)}, \quad i=1,2,
\end{aligned}
$$




$$
\begin{aligned}
C_{1} & \equiv \frac{c_{0}}{\varphi_{1}-\varphi_{2}}\left[\left(\bar{M}_{\infty}-\bar{\zeta} c_{1} c_{2}\right) \varphi_{2}-c_{3}\left(\bar{M}_{\infty}-\bar{\zeta} c_{1} c_{4}\right)\right], \\
C_{2} & \equiv \frac{c_{0}}{\varphi_{1}-\varphi_{2}}\left[c_{3}\left(\bar{M}_{\infty}-\bar{\zeta} c_{1} c_{4}\right)-\varphi_{1}\left(\bar{M}_{\infty}-\bar{\zeta} c_{1} c_{2}\right)\right] .
\end{aligned}
$$

Letting $\bar{t} \rightarrow \infty$ in Eqs. (18a) and (18b) leads to the following steady-state values of the coating stresses and the curvature:

$$
\begin{aligned}
& \bar{\sigma}_{c}^{\mathrm{bot}}(\infty)=-\varepsilon_{\infty}^{*} \bar{M}_{\infty} \frac{1+\left(3 \beta^{2}+4 \beta^{3}\right) \bar{M}_{\infty}}{1+\left(4 \beta+6 \beta^{2}+4 \beta^{3}\right) \bar{M}_{\infty}+\beta^{4} \bar{M}_{\infty}^{2}}, \\
& \bar{\sigma}_{c}^{\mathrm{top}}(\infty)=-\varepsilon_{\infty}^{*} \bar{M}_{\infty} \frac{1-\left(3 \beta^{2}+2 \beta^{3}\right) \bar{M}_{\infty}}{1+\left(4 \beta+6 \beta^{2}+4 \beta^{3}\right) \bar{M}_{\infty}+\beta^{4} \bar{M}_{\infty}^{2}},
\end{aligned}
$$

$$
\bar{\kappa}(\infty)=\varepsilon_{\infty}^{*} \bar{M}_{\infty} \frac{6 \beta(1+\beta)}{1+\left(4 \beta+6 \beta^{2}+4 \beta^{3}\right) \bar{M}_{\infty}+\beta^{4} \bar{M}_{\infty}^{2}} .
$$

Note that Eq. (20c) is consistent with published generalizations of Stoney's formula for thick elastic coatings, ${ }^{7,13,31}$ provided that the coating is assigned a biaxial modulus of $M_{\infty}$.

As can be seen from Eq. (19a), the form of solution is degenerate for the case of $\bar{\tau}=1$, in which case appropriate limiting processes must be performed on Eqs. (18a) and (18b). (See Appendix A.)

The functional dependence of the exact solution indicates that the normalized coating stresses and curvature, if further scaled by the steady-state eigenstrain $\varepsilon_{\infty}^{*}$, depend on four parameters: $\beta, \bar{M}_{0}, \bar{M}_{\infty}$, and $\bar{\tau}$. This dependence will be explored in detail later in the paper.

\section{Rapid-eigenstrain solution $(\bar{\tau}=0)$}

The rapid-eigenstrain solution may be obtained by taking the $\bar{\tau} \rightarrow 0$ limit of the general solution [Eqs. (18) and (19)], in which case the time-dependence of the response is due solely to the coating relaxation. As a result, both the coating stresses and the curvature instantaneously reach their maxima at time zero, and these maxima correspond to an initial elastic response; thereafter, the response decreases monotonically as the coating relaxes.

For fixed values of $\beta, \bar{M}_{0}, \bar{M}_{\infty}$, and $\varepsilon_{\infty}^{*}$, the rapideigenstrain case represents an extreme-case scenario. More specifically, the maximum response (stress, strain, curvature, or deflection) of the $\bar{\tau}=0$ solution will exceed that for any case in which $\bar{\tau}>0$. Thus, the rapid-eigenstrain solution provides a means for determining a simple upper bound on the exact value of the OSR for an arbitrary value of $\bar{\tau}$.

$$
\mathrm{OSR}_{\text {exact }} \equiv \frac{\kappa_{\max }}{\kappa(\infty)} \leqslant\left.\mathrm{OSR}\right|_{\bar{\tau} \rightarrow 0},
$$

where

$$
\left.\operatorname{OSR}\right|_{\bar{\tau} \rightarrow 0}=\frac{1+\left(4 \beta+6 \beta^{2}+4 \beta^{3}\right) \bar{M}_{\infty}+\beta^{4} \bar{M}_{\infty}^{2}}{1+\left(4 \beta+6 \beta^{2}+4 \beta^{3}\right) \bar{M}_{0}+\beta^{4} \bar{M}_{0}^{2}} \frac{\bar{M}_{0}}{\bar{M}_{\infty}}
$$

Because $M_{\infty} \leqslant M_{0}$, the expression on the right-hand side of Eq. (21b) is bounded above by $\bar{M}_{0} / \bar{M}_{\infty}=M_{0} / M_{\infty}$ (property of the coating material), regardless of the value of $\beta$. Inequality (21a) and Eq. (21b) provide a potentially useful theoretical upper limit on the overshoot magnitude of the coated-beam's response. In addition, the simple analytical result of Eq. (21b) is an exact result for the rapid-eigenstrain case, is valid for arbitrary coating thickness and arbitrary initial and asymptotic moduli of the coating, and can serve as an accurate approximation of the exact OSR for small, but nonzero, values of $\bar{\tau}$.

\section{Thin-coating solution}

\section{First-order approximate solution}

While numerical results may easily be generated from the exact solution for a general exponential eigenstrain, the complexity of the solution form may hide relatively simple relationships that exist in cases of practical interest. In particular, for MC-based chemical sensor applications the viscoelastic (e.g., polymer) coating is often relatively thin in comparison with the elastic (e.g., silicon) base layer. Therefore, a thin-coating approximation for the beam response will be pursued. Such an approximate solution may be obtained by expanding the exact solution in powers of $\beta$ and ignoring higher-order terms.

In this section approximate results for both the curvature and stress histories will be presented. These results will be denoted as a "first-order solution" because all terms of order $\beta^{n}, n>1$, will be ignored in the curvature expansion. For consistency, the order of the corresponding approximate stress will be one degree lower than that of the curvature, as indicated by Eq. (18c). Thus, the first-order solution will involve an $O(\beta)$ curvature expression [with $O\left(\beta^{2}\right)$ error] and stress expressions of $O(1)$ accuracy [with $O(\beta)$ error]. Expansions of Eqs. (18a)-(18c) result in the desired first-order solution:

$$
\begin{aligned}
& \bar{\sigma}_{c}^{\mathrm{bot}}(\bar{t}) \approx \bar{\sigma}_{c}^{\mathrm{top}}(\bar{t}) \approx-\varepsilon_{\infty}^{*}\left[\bar{M}_{\infty}\left(1-e^{-\bar{t}}\right)+\bar{\zeta}\left(e^{-\bar{t}}-e^{-\bar{t} / \bar{\tau}}\right)\right], \\
& \bar{\kappa}(\bar{t}) \approx 6 \beta \varepsilon_{\infty}^{*}\left[\bar{M}_{\infty}\left(1-e^{-\bar{t}}\right)+\bar{\zeta}\left(e^{-\bar{t}}-e^{-\bar{t} / \bar{\tau}}\right)\right] .
\end{aligned}
$$

Note that this solution yields equal stresses at the top and bottom of the coating, i.e., the coating stress is approximated as uniform; thus, by Eq. (13), the neutral axis is fixed and given by $\bar{n}(\bar{t})=2 / 3$ (a result consistent with Stoney's classical analysis). The approximate formulas are degenerate when $\bar{\tau}=1$; the appropriate limits for this case are therefore included in Appendix B.

Also of note is the proportionality exhibited between coating stress and curvature in the first-order solution [Eqs. (22a) and (22b)], which is simply a time-dependent restatement of Stoney's formula $\left(\bar{\kappa}=-6 \beta \bar{\sigma}_{c}\right.$ in our notation). ${ }^{6} \mathrm{Re}-$ 
call that, in the derivation of Stoney's equation, no assumptions were made regarding the coating properties, provided that the coating is infinitesimally thin. Therefore, one should not be surprised that Stoney's equation holds pointwise in time for the case of a thin, viscoelastic coating. However, the first-order solution clearly shows how the curvature and stress evolve over time, and therefore represents a potentially useful extension of Stoney's solution for cases involving coatings that may be modeled as three-parameter solids.

\section{First-order solution with asymptotic correction}

The first-order solution may be improved by recognizing that the exact steady-state results are known and take relatively simple forms [Eqs. (20a)-(20c)]. This knowledge enables one to apply multiplicative correction factors to the first-order results, thereby ensuring that they are exact in the limit as $\bar{t} \rightarrow \infty$. The resulting approximate solution will be referred to as the "corrected first-order solution." The coating stresses of the corrected solution may be obtained by multiplying Eq. (22a) by the following correction factors to obtain, respectively, the stress histories at the bottom and top of the coating:

$$
\begin{aligned}
K_{\sigma, \text { bot }} & =\frac{1+\left(3 \beta^{2}+4 \beta^{3}\right) \bar{M}_{\infty}}{1+\left(4 \beta+6 \beta^{2}+4 \beta^{3}\right) \bar{M}_{\infty}+\beta^{4} \bar{M}_{\infty}^{2}}, \\
K_{\sigma, \text { top }} & =\frac{1-\left(3 \beta^{2}+2 \beta^{3}\right) \bar{M}_{\infty}}{1+\left(4 \beta+6 \beta^{2}+4 \beta^{3}\right) \bar{M}_{\infty}+\beta^{4} \bar{M}_{\infty}^{2}} .
\end{aligned}
$$

The curvature of the corrected first-order solution is given by multiplying Eq. (22b) by

$$
K_{\kappa}=\frac{1+\beta}{1+\left(4 \beta+6 \beta^{2}+4 \beta^{3}\right) \bar{M}_{\infty}+\beta^{4} \bar{M}_{\infty}^{2}} .
$$

Because all correction factors are $O(1)$ as $\beta \rightarrow 0$, their application to the (uncorrected) first-order solution does not alter the order of the error terms. Also note that, prior to applying the correction factors, the first-order solution predicts uniform coating stresses; however, the corrected solution yields unequal stresses at the top and bottom of the coating, i.e., a nonuniform coating stress distribution, which is consistent with the linear distribution exhibited by the exact solution.

\section{Overshoot criterion/characteristics}

The qualitative behavior of the theoretical beam response due to an exponential eigenstrain may be classified as one of two types. In some cases the response monotonically increases with time, while in others it exhibits an overshoot phenomenon. The observation of both types of response signatures in MC sensor data (e.g., Fig. 1) ${ }^{1,26,27}$ provides the motivation to seek a simple mechanics-based criterion by which the response type may easily be predicted. In addition, such a criterion could be useful in (a) extracting a system parameter from a response signature or (b) in the case of sensor applications, correlating transient response characteristics to ambient analyte concentration. In this section a simple overshoot criterion will be derived for predicting the response type, as will expressions for the OSR and the "critical time" at which the peak response occurs.

Due to the complexity of the exact solution, derivation of an exact overshoot criterion is not feasible. However, the first-order approximate solution is relatively simple and, thus, conducive to the development of a corresponding overshoot criterion. A straightforward analysis of the first-order solution [Eq. (22b) or its "corrected" counterpart] leads to the overshoot criterion summarized below. While this criterion will, strictly speaking, only be valid for relatively thin coatings, it may also provide a useful guideline for understanding the behavior of systems with thicker coatings.

Overshoot criterion (based on first-order solution): Overshoot (in the curvature or stress response) occurs if and only if the coating may experience relaxation (i.e., $M_{\infty} / M_{0}$ $<1$ ) and

$$
\vartheta \equiv \frac{1}{\bar{\tau}} \frac{M_{0}}{M_{\infty}}>1,
$$

where $\vartheta$ is deemed the "overshoot parameter."

When the value of the overshoot parameter is unity, the system response is on the "boundary" between overshoot and monotonic responses. Values greater than unity correspond to overshoot behavior, with larger values representing more significant overshoot. Thus, parameter $\vartheta$ may be interpreted as a figure of merit with regard to the tendency of a particular coated beam to exhibit overshoot in response to a particular exponential coating eigenstrain history.

When overshoot does occur, a straightforward analysis of Eq. (22a) or Eq. (22b) leads to an expression for estimating the critical time at which the maximum stress or curvature occurs:

$$
\bar{t}_{\mathrm{cr}} \approx \frac{\bar{\tau}}{\bar{\tau}-1} \ln \left(\frac{\bar{\tau} \vartheta-1}{\vartheta-1}\right) .
$$

Placing Eq. (25) into Eq. (22b) and scaling by Eq. (20c) yields an estimate of the OSR:

$$
\mathrm{OSR} \approx 1-e^{-\bar{t}_{\mathrm{cr}}}+(\vartheta-1) \frac{\bar{\tau}}{1-\bar{\tau}}\left(e^{-\bar{t}_{\mathrm{cr}}}-e^{-\bar{t}_{\mathrm{cr}} / \bar{\tau}}\right)
$$

(The same expression is applicable to a stress-based OSR.) This form is degenerate for $\bar{\tau}=0$ or $\bar{\tau}=1$, in which cases the appropriate limit must be taken. For the rapid-eigenstrain case $(\bar{\tau}=0)$, Eq. (26) reduces to the following simple firstorder estimate applicable to thin coatings:

$$
\left.\mathrm{OSR}\right|_{\bar{\tau} \rightarrow 0} \approx \frac{M_{0}}{M_{\infty}}
$$

which also could have been derived directly from the exact expression for the OSR, Eq. (21b). The limiting result for the $\bar{\tau}=1$ case is included in Appendix B.

Several comments are in order regarding the overshoot criterion and overshoot characteristics. First, if one recognizes that the reciprocal of the relative time constant, $1 / \bar{\tau}$, may be viewed as a "relative eigenstrain rate" and defines $M_{\infty} / M_{0}$ to be the "relaxation ratio" of the coating, then the 
overshoot criterion given by inequality (24) may be written as

$$
\frac{1}{\bar{\tau}}>\frac{M_{\infty}}{M_{0}}
$$

and interpreted as follows: overshoot will occur if and only if the relative eigenstrain rate is larger than the relaxation ratio of the coating. Thus, the overshoot phenomenon is governed by the relative magnitude of the two competing rates-the eigenstrain rate and the coating relaxation rate. In particular, if the coating material is capable of full relaxation $\left(M_{\infty} / M_{0}\right.$ $=0$ ), the response will exhibit overshoot regardless of the relative eigenstrain rate. This is to be expected since the steady-state curvature in this case must be zero because the fully relaxed coating cannot transfer stress to the base layer; therefore, any transient curvature that occurs prior to complete relaxation of the coating must necessarily exceed the zero steady-state value.

The overshoot criterion may be written in an even simpler form if one notes that the creep time constant $\tau_{C}$ of the coating material (corresponding to the strain history caused by a step-function biaxial stress) is related to the relaxation time constant $\tau_{R}$ by ${ }^{19}$

$$
\tau_{C}=\frac{M_{0}}{M_{\infty}} \tau_{R},
$$

which means that the overshoot criterion (24) may be expressed as

$$
\vartheta \equiv \frac{\tau_{C}}{\tau_{\varepsilon}}>1,
$$

i.e., overshoot is governed by the relative magnitudes of the creep and eigenstrain time constants of the coating.

In examining Eqs. (25) and (26), one notices that the first-order results for $\bar{t}_{\mathrm{cr}}$ and the OSR depend only on $\bar{\tau}$ and $M_{\infty} / M_{0}$. This implies that, when a MC sensor with a thin selective coating experiences exponential analyte sorption, the values of $\bar{t}_{\mathrm{cr}}$ and OSR depend only on the coating/analyte pair, not on the system geometry or the base material's properties. This result could therefore be useful in selecting a coating to obtain desirable transient response characteristics for detecting a particular analyte. Correlation of the transient signature with analyte concentration could minimize detection times by eliminating the need to wait for the steady-state signal. A similar idea has been successfully employed by others for metal-coated (palladium) MC sensors. In particular, the transient bending rate of the monotonic response was correlated to steady-state deflection and hydrogen concentration. $^{32}$

Finally, one should note that the OSR estimate [Eq. (26)] takes an even simpler form [Eq. (27)] when $\bar{\tau}=0$, which provides an upper bound on Eq. (26). Equations (27) and (28) show that, within the context of first-order (thin-coating) theory, the value of the coating property $M_{0} / M_{\infty}$ furnishes two important response characteristics of the coated beam. Its value provides (a) the OSR for the rapid-eigenstrain $(\bar{\tau}$ $=0$ ) case, which is an upper bound on the OSR for other $\bar{\tau}$ values, and (b) the "transitional" value of $\bar{\tau}$ that separates overshoot response from monotonic behavior. These observations may provide a theoretical basis for extracting the biaxial relaxation ratio of a thin coating from the coatedbeam response signature. An analogous approach has been implemented to deduce the biaxial elastic modulus and coefficient of thermal expansion of thin elastic films by measuring the thermal deformation of coated beams. ${ }^{33}$

\section{NUMERICAL RESULTS AND DISCUSSION}

Numerical results for the case of exponential coating eigenstrain will now be presented. Results corresponding to the exact and thin-coating solutions will be included. For all of the time-history plots presented, dimensionless quantities will be utilized in order to increase the generality of the results. Normalized time-histories of both curvature (deflection) and stress will be plotted for fixed values of the four dimensionless parameters, $\beta \equiv h_{2} / h_{1}, \quad \bar{M}_{0} \equiv M_{0} / M, \quad \bar{\tau}$ $\equiv \tau_{\varepsilon} / \tau_{R}$, and a new parameter called the coating's "relative relaxation parameter" $\rho$, defined by

$$
\rho \equiv \frac{M_{0}-M_{\infty}}{M_{0}}=1-\frac{M_{\infty}}{M_{0}}=1-\frac{\bar{M}_{\infty}}{\bar{M}_{0}} .
$$

While parameter $\bar{M}_{\infty}$ appeared "naturally" in the mathematical formulation as the fourth system parameter [see, e.g., Eq. (9)], parameter $\rho$ is a more convenient choice for presenting results because (a) it depends only on the coating material, (b) its values are confined to the range $[0,1]$, and (c) it has a simple physical meaning: it represents the percent relaxation that is possible in the coating, i.e., $\rho=0.25$ denotes $25 \%$ relaxation capability.

In order to limit the number of figures, all results will correspond to the following input values unless stated otherwise: $\beta=0.1$ and 0.5 (thin and thick coatings); $\bar{M}_{0}=0.1$ (relatively flexible coating material); $\rho=0.1$ and 0.5 (10\% and $50 \%$ coating relaxation); and $\bar{\tau}=0,0.1,0.5,1,2$, and 5 . Thus, the $\bar{\tau}$ values range from the rapid eigenstrain (or slow relaxation) case to a very slow eigenstrain (or rapid relaxation) case. Results may easily be generated for other parameter values.

Exact results for beam deformation. The exact timehistories of the beam deformation, shown in Figs. 5(a)-5(d), were generated using Eqs. (18) and (19) and, in the $\bar{\tau}=1$ case, Eqs. (A1a) and (A1b). As expected, the curvature is positive (downward tip deflection) when the eigenstrain is positive (extensional). Also of note is that all curves in each figure approach the same steady-state value, but at different rates depending on the $\bar{\tau}$ value. For a given $\beta$ value, the steady-state curvature depends on the relative relaxation parameter $\rho$, and its value corresponds to that predicted by an elastic model that utilizes a coating modulus equal to the relaxed modulus of the viscoelastic material. [See Eq. (20c).] Of course, for a coating material that may experience full relaxation ( $\rho=1$, not shown), the asymptotic curvature would be zero.

Also observed in Figs. 5(a)-5(d) is an overshoot for particular values of $\bar{\tau}$. As $\bar{\tau}$ decreases, overshoot becomes more prominent. The first-order overshoot criterion is able to ac- 


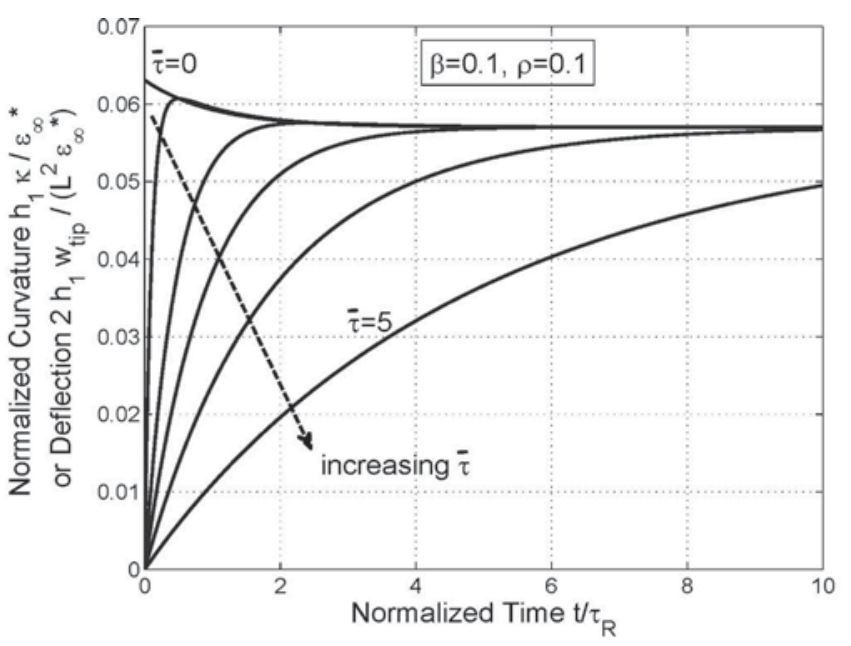

(a)

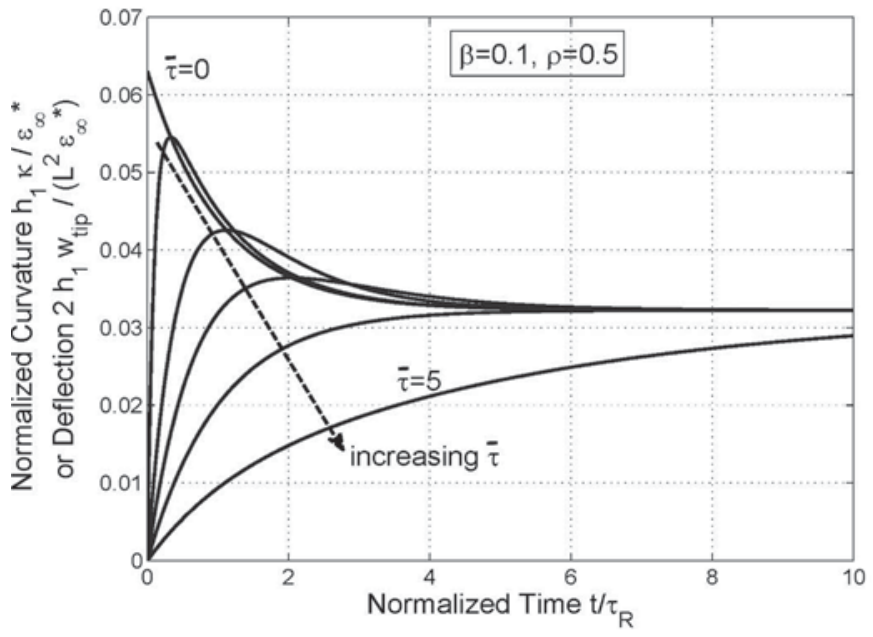

(b)

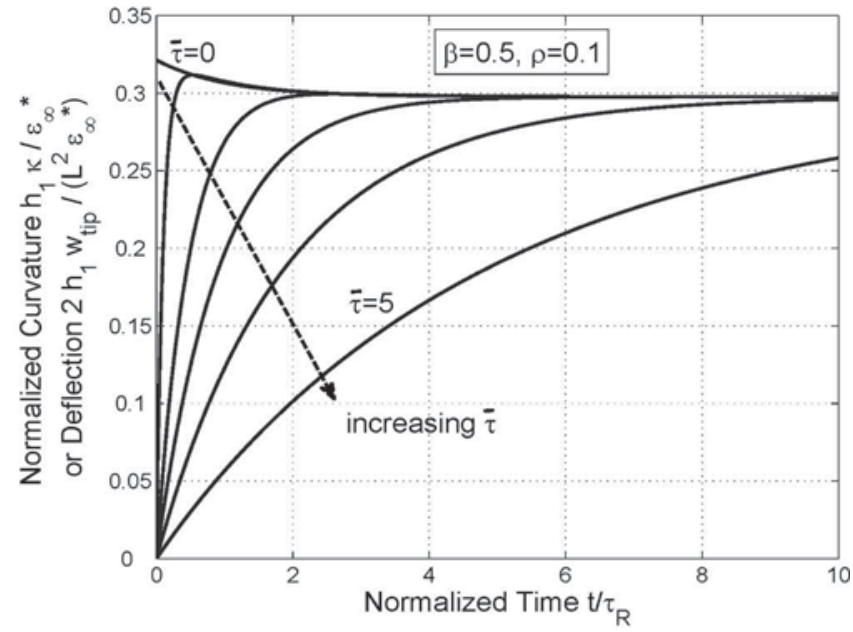

(c)

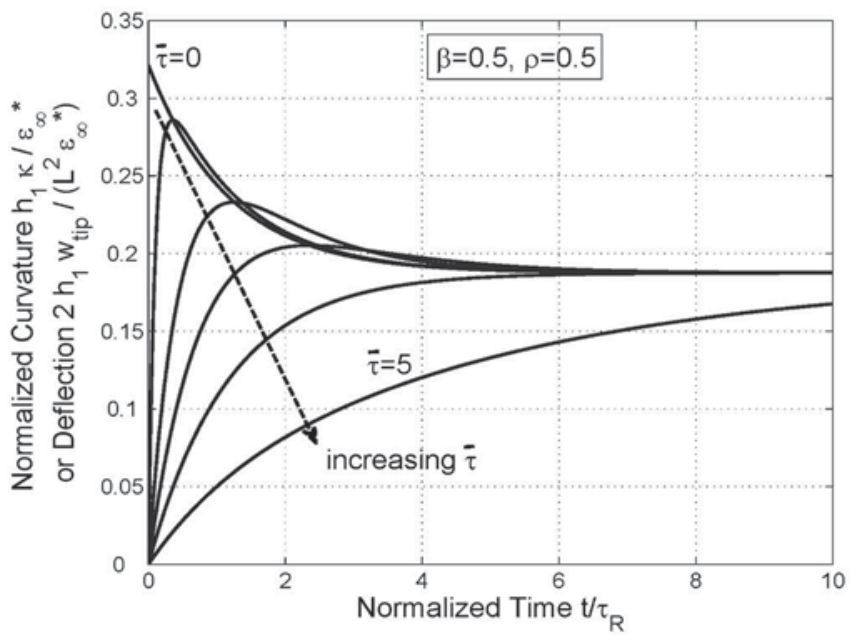

(d)

FIG. 5. Exact response vs time for exponential eigenstrain history: $\bar{M}_{0}=0.1$ and $\bar{\tau}=[0,0.1,0.5,1.0,2.0,5.0]$.

curately predict which of the exact curves exhibit overshoot. For the chosen $\rho$ values of 0.1 and 0.5 , the criterion predicts that the transitional values of $\bar{\tau}$ that separate overshoot behavior from monotonic behavior are 1.11 and 2.0, respectively. All curves having $\bar{\tau}$ values less than (greater than) the corresponding transitional value do indeed exhibit overshoot (monotonic) behavior. (In the $\bar{\tau}=1$ case of Fig. 5(a), a relative maximum occurs just beyond $\bar{t}=10$; the peak, however, is barely perceptible because the response is very close to the transition from overshoot to monotonic behavior.) Thus, although the overshoot criterion was based on a thin-coating (first-order) approximation, it can also serve as an accurate guideline even for thicker coatings ( $\beta=0.5$ in this case).

The timing of the overshoot in Figs. 5(a)-5(d) follows a clear trend: as $\bar{\tau}$ decreases the overshoot peak occurs earlier, approaching time zero as $\bar{\tau} \rightarrow 0$. The $\bar{\tau}=0$ response displays the expected behavior of the rapid-eigenstrain solution: the response begins with a sudden elastic curvature, followed by a gradual decrease toward steady state as the coating relaxes.
Comparing the deformation magnitudes in Figs. 5(a) and 5(b) with those in Figs. 5(c) and 5(d), one sees that, as the thickness ratio $\beta$ increases fivefold, the magnitude of the deformation increases by approximately the same factor. This was to be anticipated given the form of the first-order solution [Eq. (22b)], which indicates that the deformation history is approximately linear in $\beta$ for small $\beta$, with the higher-order effects being relatively insignificant.

Exact results for coating stresses. Plots of the exact coating stresses [Eqs. (18a) and (18b)] are shown in Figs. 6(a)-6(d). As expected, the coating stresses are compressive for the case of a positive (extensional) eigenstrain due to the restraining effect of the base layer on the coating expansion. The stress at the interface (bottom of the coating), where the restraining effect is most pronounced, has a larger magnitude than that at the top. Also, on each figure all the curves for the coating stress at the top approach the same asymptotic value at large times, as do those for the bottom coating stresses (although not the same asymptotic value). The value of $\bar{\tau}$ 


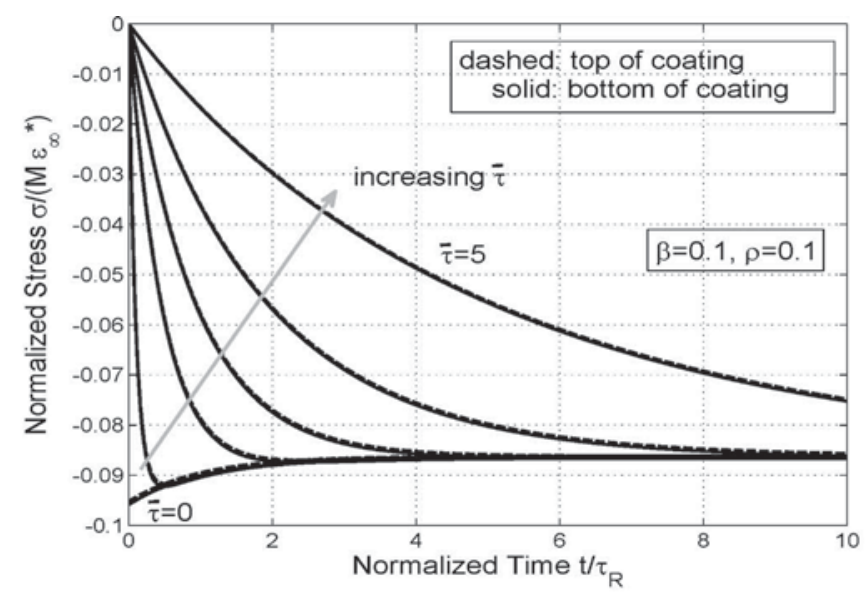

(a)

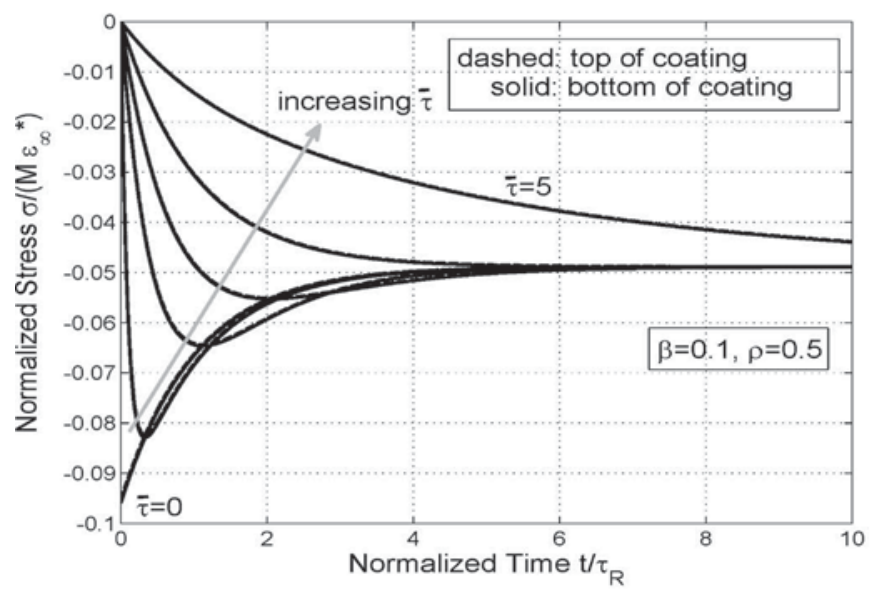

(b)

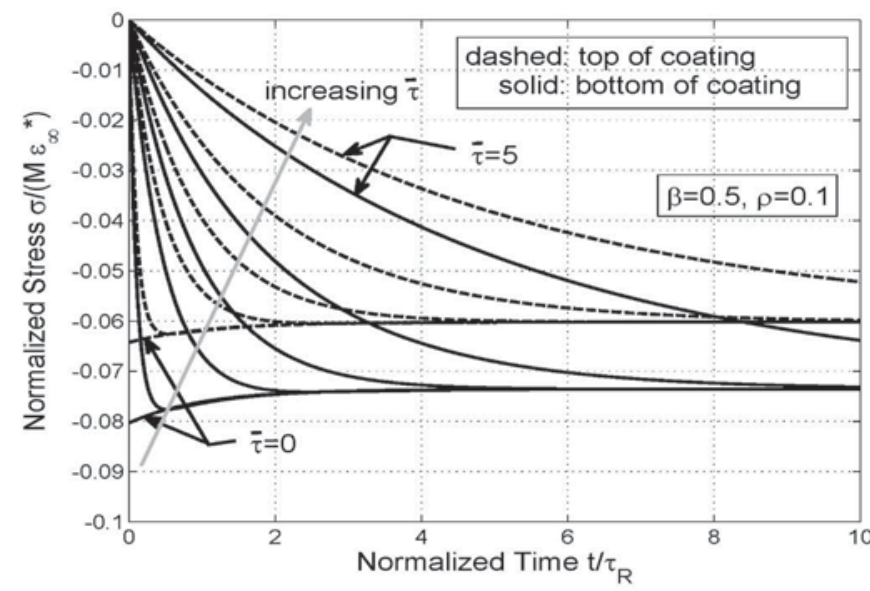

(c)

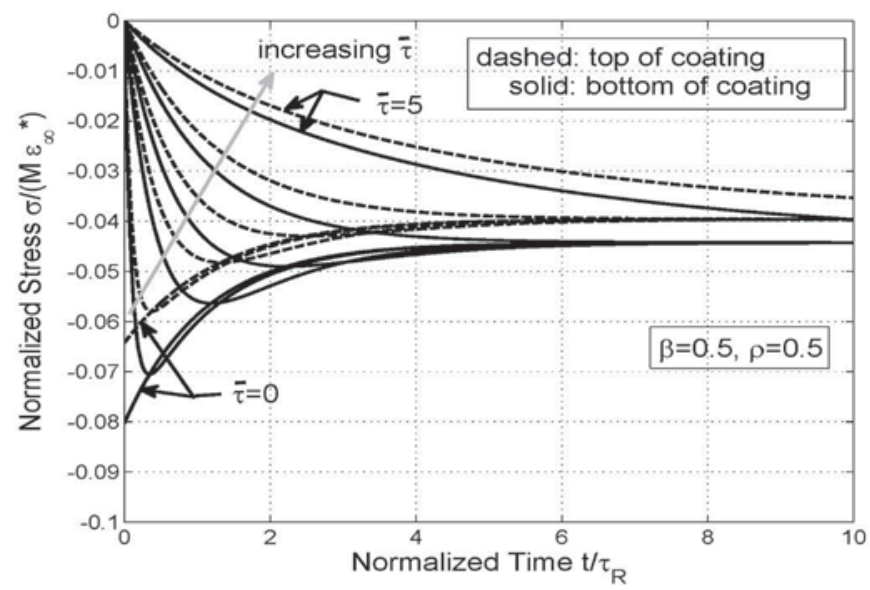

(d)

FIG. 6. Exact coating stresses vs time for exponential eigenstrain history: $\bar{M}_{0}=0.1$ and $\bar{\tau}=[0,0.1,0.5,1.0,2.0,5.0]$.

affects the rate at which the steady-state value is approached. Similar to what was noted for beam deformation, the steadystate values of coating stress would be zero for the case of $\rho=1$ because a fully relaxed coating is incapable of sustaining stress. The existence of overshoot in the stress histories is dictated by the value of $\bar{\tau}$ and, as was true for the curvature, the first-order overshoot criterion is able to predict if overshoot exists in the stress histories. All previous comments regarding curvature overshoot also apply to the stress histories.

The plots in Figs. 6(a) and 6(b) illustrate that the stresses at the top and bottom of the coating are essentially identical for the relatively thin coating $(\beta=0.1)$, i.e., the coating stress is uniform. However, for the thicker coating case $(\beta=0.5)$ of Figs. 6(c) and 6(d), the coating stress is clearly nonuniform as indicated by the different values of stress at the top and bottom of the coating. Comparing the results of these latter two figures shows that, as $\rho$ increases (more coating relaxation), the stresses tend to become more uniform throughout the coating. Also noteworthy is that, unlike the curvature magnitude, the coating stress magnitude does not change significantly as $\beta$ changes from 0.1 to 0.5 . This is a reflection of the fact that the leading term of the coating stress expansion is constant ( $\beta$-independent) while the leading term in the curvature series is linear in $\beta$. [Compare Eqs. (22a) and (22b).]

Approximate versus exact beam deformation history. In order to explore the accuracy of the first-order solutions (uncorrected and corrected), the exact and approximate curvature histories are plotted in Figs. 7(a) and 7(b) for a thin coating $(\beta=0.1)$ and in Figs. $7(\mathrm{c})$ and $7(\mathrm{~d})$ for a thick coating $(\beta=0.5)$. All of these figures correspond to a coating for which $\rho=0.5$. For both the thin- and thick-coating cases, two values of $\bar{\tau}$ have been considered: $\bar{\tau}=0.5$, representing relatively fast eigenstrain causing overshoot, and $\bar{\tau}=5$, corresponding to a slow eigenstrain process and, thus, a monotonic response. All four figures indicate that the uncorrected thin-coating solution underestimates the exact curvature. However, when the asymptotic correction factor is applied, the vast majority of the error is removed throughout the en- 


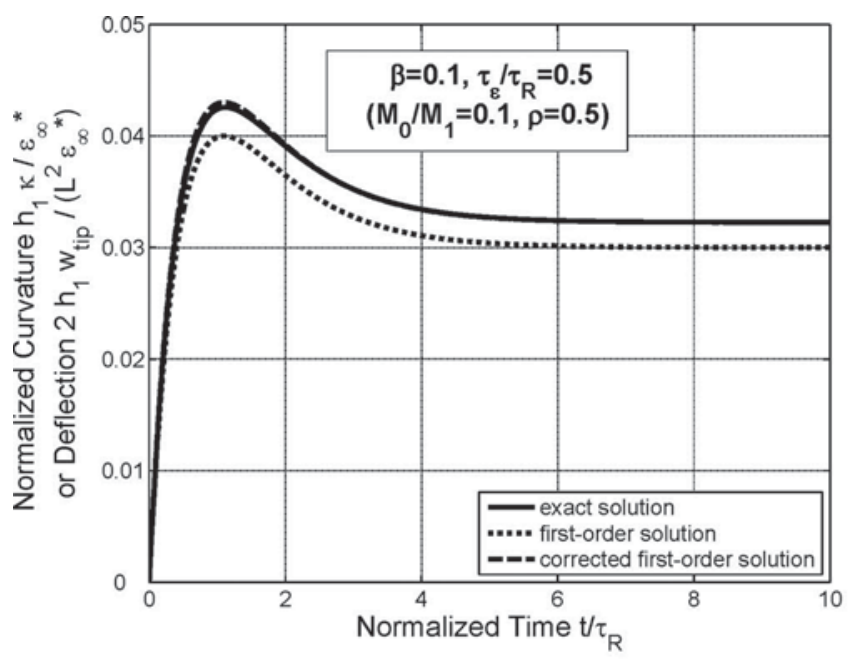

(a)

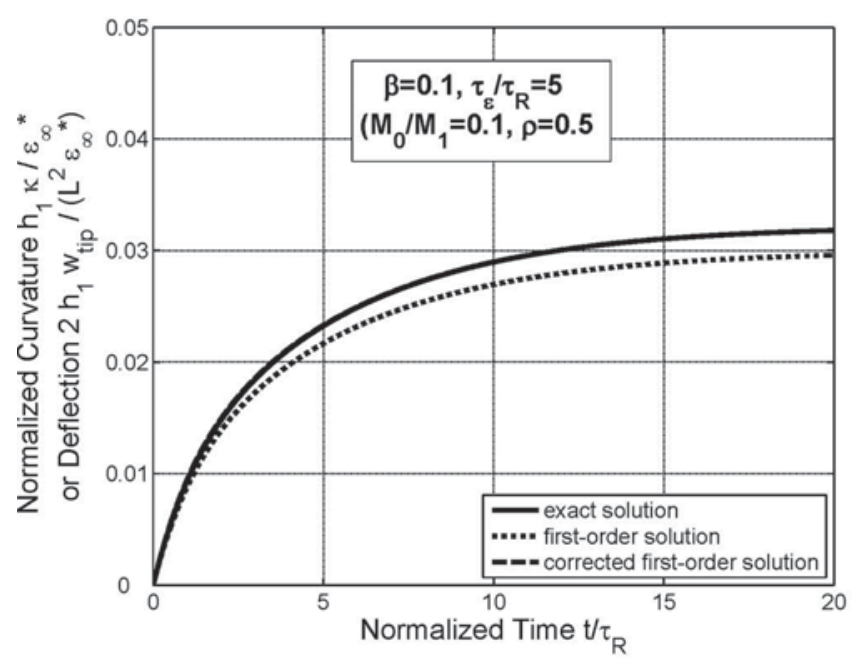

(b)

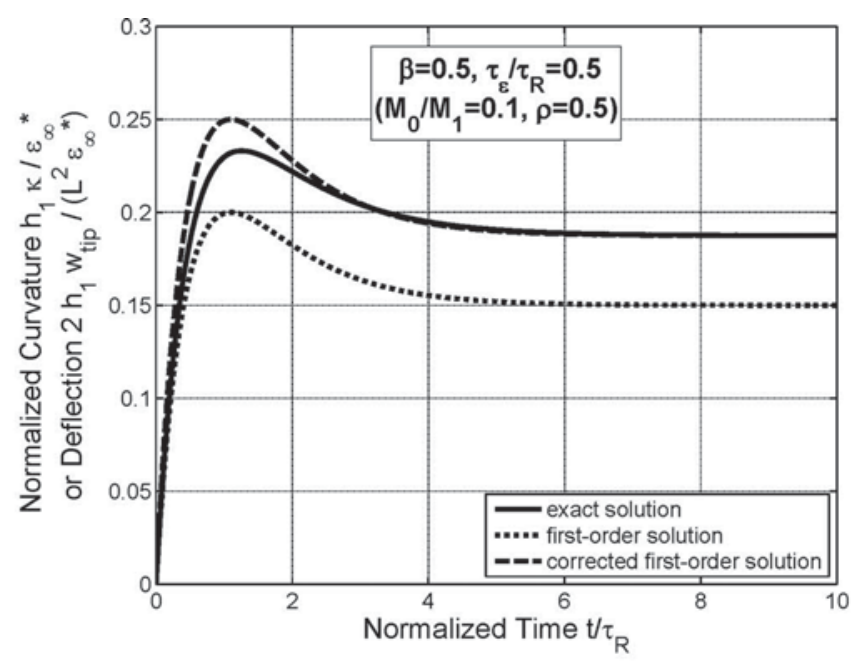

(c)

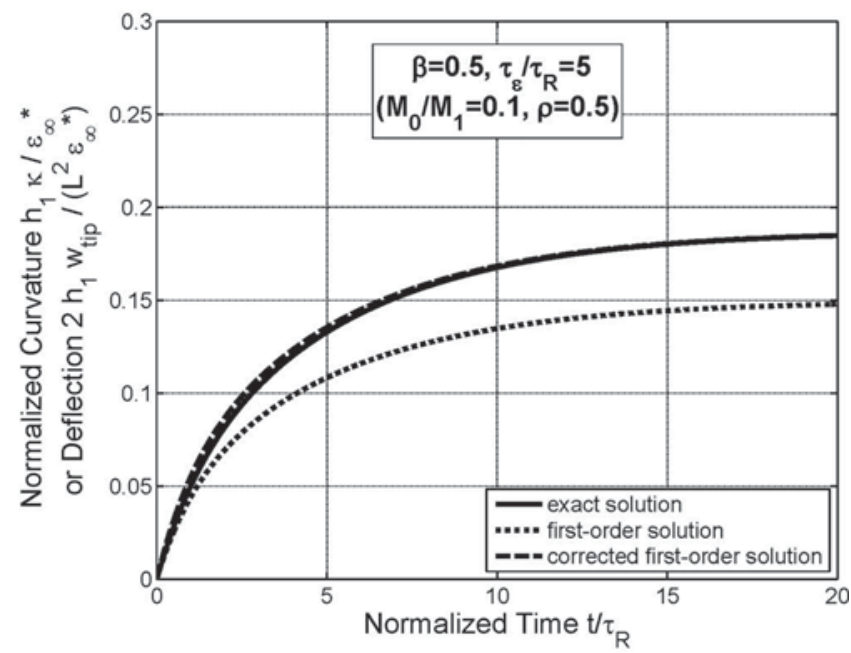

(d)

FIG. 7. Comparisons of exact and approximate responses for exponential eigenstrain history: $\bar{M}_{0}=0.1$ and $\rho=0.5$ in all cases.

tire range of the response. For the thin coating [Figs. 7(a) and 7(b)] the corrected solutions are essentially exact. As expected, the accuracy of the approximate solution decreases as the coating thickness is increased [Figs. 7(c) and 7(d)] and the uncorrected solution exhibits significant departure from the exact response. However, the corrected solution shows marked improvement. When the response is monotonic [Fig. 7(d)], the corrected solution is virtually exact, even for the thick coating case. When the response includes overshoot [Fig. 7(c)], the corrected solution still contains some residual error that is concentrated near the response peak, resulting in an overestimation of the exact response. Nevertheless, the corrected solution is still quite good.

A quantitative comparison of the errors of the approximate solutions in Figs. 7(a)-7(d) is given in Table I. The error metric involves normalization with respect to the maximum value of the exact curvature instead of the local exact value. This definition avoids indeterminate relative errors at time zero (where all approximations are exact). The tabulated data show that the corrected first-order solution yields errors of no more than $10 \%$ in all cases considered. For more flexible coatings $\left(\bar{M}_{0}<0.1\right)$ or coating relaxations less than $50 \%$ (not shown), these errors are reduced even further. (The errors would increase for stiffer coatings or coatings with more relaxation.) The tabulated results also show that the accuracy of the corrected solution decreases as the coating thickness increases or as $\bar{\tau}$ decreases. Although not included here, figures comparing the exact and approximate coating

TABLE I. Maximum normalized error in curvature for the approximate solutions in Figs. $7(\mathrm{a})-7(\mathrm{~d})\left(\bar{M}_{0}=0.1, \rho=0.5\right)$. The "normalized error" is defined as the magnitude of the absolute error in the curvature (lexact -approx.|) divided by the maximum value of the exact curvature.

\begin{tabular}{cccc}
\hline \hline$\beta$ & $\bar{\tau}$ & $\begin{array}{c}\text { First-order } \\
(\%)\end{array}$ & $\begin{array}{c}\text { Corrected first-order } \\
(\%)\end{array}$ \\
\hline \multirow{2}{*}{0.1} & 0.5 & 6.2 & 1.4 \\
& 5 & 6.9 & 0.4 \\
0.5 & 0.5 & 17.4 & 10.0 \\
& 5 & 19.7 & 2.9 \\
\hline
\end{tabular}




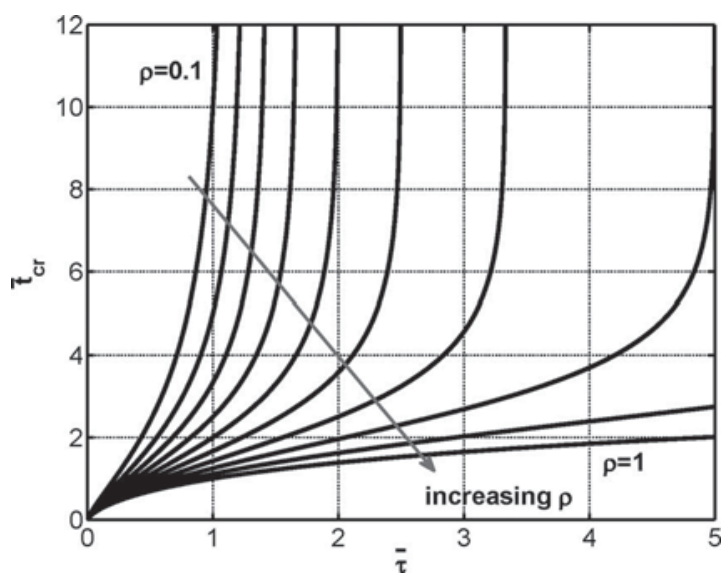

FIG. 8. Time of peak overshoot response vs $\bar{\tau}$, exponential eigenstrain history (first-order theory); increment in $\rho$ is 0.1 .

stresses lead to similar conclusions as did the curvature comparisons regarding the accuracy of the uncorrected and corrected thin-coating stress formulas.

First-order estimate for $\bar{t}_{\mathrm{cr}}$. When the coating thickness is sufficiently small, the analytical result [Eq. (25)] may be used to obtain an accurate estimate of the critical time $\bar{t}_{\mathrm{cr}}$ in terms of only two dimensionless parameters, $\bar{\tau}$ and $\rho$, i.e., the time of the peak response is independent of $\beta$ and $\bar{M}_{0}$. Using Eqs. (24), (25), and (31) enables one to obtain the desired analytical expression for $\bar{t}_{\mathrm{cr}}$, the results of which are shown in Fig. 8. The curves clearly show that the peak response occurs earlier as $\bar{\tau}$ decreases or $\rho$ increases. Thus, for a fixed value of $\tau_{R}$, either of the following will result in an earlier peak response: (a) an increase in the coating eigenstrain rate (decrease in $\tau_{\varepsilon}$ ) or (b) an increase in the amount of stress relaxation in the coating. Each curve displayed in Fig. 8 approaches a vertical asymptote at the transitional value of $\bar{\tau}$ given by $(1-\rho)^{-1}$ (or $\left.M_{0} / M_{\infty}\right)$, which corresponds to the overshoot parameter $\vartheta$ being equal to 1 ; as a result, any value of $\bar{\tau}$ in excess of this transitional value corresponds to a monotonic response, i.e., $\bar{t}_{c r} \rightarrow \infty$.

Because the results of Fig. 8 are approximations based on first-order theory, a few comments are in order concerning their accuracy. A detailed examination of exact curvature histories indicates that the $\bar{t}_{\text {cr }}$ values in Fig. 8 provide lower bounds to the exact results, i.e., the exact peak response occurs later than the time predicted by Fig. 8. However, in many cases of practical interest, the difference is quite small. In particular, the relative error magnitude for the $\bar{t}_{\mathrm{cr}}$ values of Fig. 8 (versus the exact values) will not exceed $5 \%$ provided that $\beta \leqslant 0.2, \bar{M}_{0} \leqslant 0.1, \rho \leqslant 0.5$, and $\bar{\tau} \leqslant 0.5 /(1-\rho)$. The latter inequality states that $\bar{\tau}$ lies in the lower half of the overshoot range of $\bar{\tau}$.

First-order estimate for OSR. The dependence of the OSR on the system and load parameters will now be examined, as will the accuracy of the simple first-order estimate [Eq. (26)]. In Fig. 9 both the exact and approximate values of the OSR are plotted versus $\bar{\tau}$ for thin and thick coatings and for $10 \%, 30 \%$, and $50 \%$ coating relaxations. As noted earlier, when $\bar{\tau}$ increases beyond a particular value, the response is monotonic and, thus, the $\mathrm{OSR}=1$. The figure illustrates that

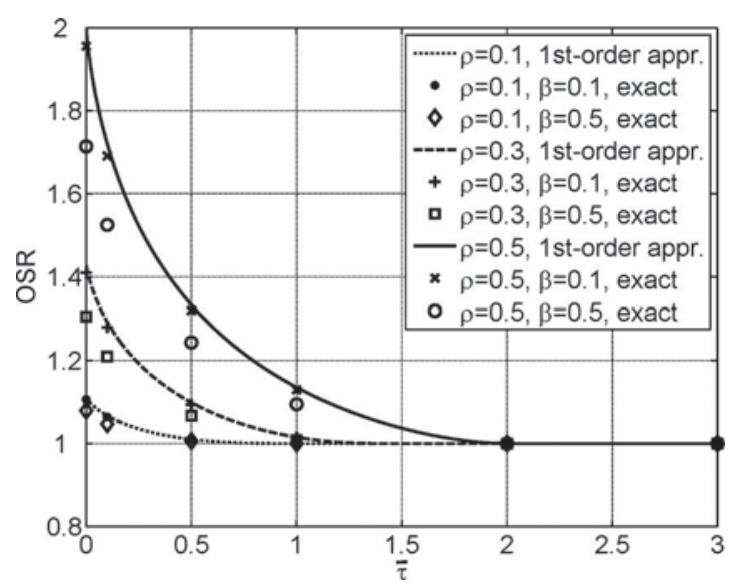

FIG. 9. OSR vs $\bar{\tau}$, exponential eigenstrain history: Comparison of exact and first-order models. Exact results based on $\bar{M}_{0}=0.1$.

the OSR increases as $\bar{\tau}$ decreases, as $\rho$ increases, and (to a lesser extent) as $\beta$ decreases, reflecting in a more succinct manner the trends that were observed earlier in the timehistory plots. Also apparent is that the simple analytical estimate for the OSR gives an excellent result in the thincoating case $(\beta=0.1)$ for the coating relaxations considered, exceeding the exact OSR by no more than $2.3 \%$, regardless of the rate at which the eigenstrain occurs. For the thick coating $(\beta=0.5)$, the approximate formula overestimates the exact OSR, with the error magnitude tending to increase as $\rho$ increases and as $\bar{\tau}$ decreases. This results in a maximum relative error of $17 \%$ for $\rho=0.5$ and $\bar{\tau}=0$. Therefore, for coatings that are thick and/or have a large amount of relaxation, the first-order OSR formula should be used judiciously in estimating the OSR value. Also, the accuracy of the firstorder formula is expected to decrease as stiffer coatings $\left(\bar{M}_{0}>0.1\right)$ are considered.

Rapid-eigenstrain formula for OSR. For those cases in which coating eigenstrain occurs very quickly (small $\bar{\tau}$ ), one need not resort to using the first-order OSR formula. Recall that a simple exact OSR formula was derived for the $\bar{\tau} \rightarrow 0$ limiting case, and this formula is applicable for arbitrary values of coating thickness and material properties. Moreover, extensive calculations have confirmed that, for all $\beta$ $\leqslant 1$ and $\bar{M}_{0} \leqslant 1$, the rapid-eigenstrain formula [Eq. (21b)] will yield results within $5 \%$ of the exact OSR for $\rho \leqslant 0.5$ and within $10 \%$ for arbitrary coating relaxation $(\rho \leqslant 1)$, provided that $\bar{\tau}<0.02$. Hence, this formula can be used over a very broad range of system parameters to predict the OSR when the eigenstrain rate (e.g., analyte sorption rate in $\mathrm{MC}$ sensor applications) is much higher than the relaxation rate of the coating material.

\section{SUMMARY, CONCLUSIONS, AND FUTURE WORK}

The initial-value problem governing the quasistatic deformation of an elastic cantilever with viscoelastic (threeparameter solid) coating, subjected to an arbitrary, timedependent coating eigenstrain, has been formulated. Exact analytical expressions for the curvature, tip deflection, and coating stresses have been derived for the case in which the eigenstrain varies exponentially in time, with a step function 
being a special case. The solution is applicable for arbitrary values of coating and base layer thicknesses, coating and base layer properties, and eigenstrain time constant. In addition, simple approximate formulas for the thin-coating case have been derived, thus providing insight into the fundamental system behavior. A simple criterion was obtained for determining if the beam response will be monotonic or exhibit overshoot. Also derived were simple approximate expressions for estimating the time at which the peak response occurs and the overshoot ratio (OSR), i.e., the maximum response scaled by the steady-state response. While the study was performed with a focus on fundamental beam mechanics, the results are expected to have important applications in MC-based (bio)chemical sensors and in the experimental characterization of thin polymer coatings. The solution should also be relevant in other areas of application because of the various physical phenomena that may cause eigenstrain (e.g., temperature change, hygroscopic swelling, phase transformation, and misfit strains).

Among the major conclusions of the study are the following: (a) The exact solution yields a simple analytical result for the OSR in the rapid-eigenstrain $(\bar{\tau} \rightarrow 0)$ case. This formula gives an upper bound on the OSR for arbitrary $\bar{\tau}$ values and may be used to accurately estimate the OSR over a wide range of system parameters provided that $\bar{\tau} \leqslant 0.02$. (b) As the normalized coating thickness $\beta \rightarrow 0$, the deflection is linear in $\beta$ while the coating stress is $\beta$-independent. (c) Overshoot occurs when the value of a simple overshoot parameter is greater than 1 . This condition corresponds to the relative eigenstrain rate being greater than the relaxation ratio of the coating or, equivalently, the creep time constant of the coating material being greater than the eigenstrain time constant. Although this criterion was derived from the thincoating solution, it has been observed to provide a useful guideline even for thick coatings. (d) When overshoot occurs, the first-order theory yields simple expressions for the OSR and the normalized time of the peak response $\left(\bar{t}_{\mathrm{cr}}\right)$, and these formulas are quite accurate over practical ranges of system parameters. The expressions depend only on the coating's relative eigenstrain rate $\bar{\tau}$ and relative relaxation parameter $\rho$. Thus, within the context of sensor applications, these two response metrics depend only on the coating/analyte pair, not on the system geometry or the properties of the base material. (e) When overshoot occurs, smaller values of $\bar{\tau}$, larger values of $\rho$, or smaller values of $\beta$ correspond to more pronounced overshoot occurring earlier in time.

The results of this theoretical study provide the motivation for future studies, including (a) experimental characterization of viscoelastic properties of thin polymer coatings, especially the relaxation time constant, in various environments, (b) experimental verification of coated-beam response predictions, (c) finite element modeling to verify the accuracy of the derived beam model, especially regarding localized stresses near the interface and support and free-edge effects, ${ }^{34}$ and making appropriate modifications to the present model to incorporate such effects; ${ }^{35-37}$ (d) generalization of the model to include through-thickness variation of coating eigenstrain, analogous to the elastic model derived by Freund, ${ }^{10,12}$ and (e) extension of the model to include the effects of interfacial slip. Regarding the latter, prior work related to elastic and elastic/perfectly plastic systems may provide a useful point of departure. ${ }^{18,38}$ In addition, the form of the present solution suggests that it may be applicable to more general coated-cantilever systems whose behaviors are governed by two competing time constants (not necessarily related to coating eigenstrain or viscoelasticity). ${ }^{39}$ Examples of other mechanisms that could be accounted for by the current solution (or its extension) include growth kinetics/ molecular rearrangement in self-aligned monomers on goldcoated cantilevers ${ }^{40,41}$ and cantilever actuation using polyelectrolyte brushes. ${ }^{42}$ These applications involve relaxation phenomena that appear to contribute to observed overshoot responses in certain instances, although this behavior has yet to be placed upon a firm theoretical foundation. The solution presented herein may provide a starting point for such theoretical endeavors.

\section{ACKNOWLEDGMENTS}

The authors would like to express sincere thanks to $\mathrm{Al}$ Loui and Timothy Ratto for providing raw data, including the data appearing in Fig. 1, and to Peter Grütter for providing useful suggestions regarding more general applications of the derived model.

\section{APPENDIX A: EXACT SOLUTION FOR THE $\bar{\tau}=1$ CASE}

Letting $\bar{\tau} \rightarrow 1$ in Eqs. (18) and (19), one may show that the exact stress histories for the $\bar{\tau}=1$ case may be obtained by replacing $\bar{\zeta} c_{1} c_{2}$ and $\bar{\zeta} c_{1} c_{4}$ in Eqs. (18a) and (18b) and Eqs. (19i) and $(19 \mathrm{j})$ with the following values:

$$
\begin{aligned}
& \lim _{\bar{\tau} \rightarrow 1} \bar{\zeta} c_{1} c_{2}=\frac{3+4 \beta}{\beta^{2}} \frac{1+\left(4 \beta+6 \beta^{2}+4 \beta^{3}\right) \bar{M}_{\infty}+\beta^{4} \bar{M}_{\infty}^{2}}{1+\left(3 \beta^{2}+4 \beta^{3}\right) \bar{M}_{\infty}} \\
& \lim _{\bar{\tau} \rightarrow 1} \bar{\zeta} c_{1} c_{4}=-\frac{3+2 \beta}{\beta^{2}} \frac{1+\left(4 \beta+6 \beta^{2}+4 \beta^{3}\right) \bar{M}_{\infty}+\beta^{4} \bar{M}_{\infty}^{2}}{1-\left(3 \beta^{2}+2 \beta^{3}\right) \bar{M}_{\infty}}
\end{aligned}
$$

The associated curvature history is obtained by substituting the stresses into Eq. (18c).

\section{APPENDIX B: THIN-COATING APPROXIMATIONS FOR THE $\bar{\tau}=1$ CASE}

Letting $\bar{\tau} \rightarrow 1$ in Eqs. (22a) and (22b) leads to the following results:

$$
\begin{array}{r}
\left.\left.\bar{\sigma}_{c}^{\mathrm{bot}}(\bar{t})\right|_{\bar{\tau} \rightarrow 1} \approx \bar{\sigma}_{c}^{\mathrm{top}}(\bar{t})\right|_{\bar{\tau} \rightarrow 1} \approx \\
-\varepsilon_{\infty}^{*} \bar{M}_{\infty}\left\{1-\left[1-\left(\frac{\bar{M}_{0}}{\bar{M}_{\infty}}-1\right) \bar{t}\right] e^{-\bar{t}}\right\}, \\
\left.\bar{\kappa}(\bar{t})\right|_{\bar{\tau} \rightarrow 1} \approx 6 \beta \varepsilon_{\infty}^{*} \bar{M}_{\infty}\left\{1-\left[1-\left(\frac{\bar{M}_{0}}{\bar{M}_{\infty}}-1\right) \bar{t}\right] e^{-\bar{t}}\right\},
\end{array}
$$




$$
\left.\mathrm{OSR}\right|_{\bar{\tau} \rightarrow 1} \approx 1+\left(\frac{M_{0}}{M_{\infty}}-1\right) e^{-1 /\left(1-M_{\infty} / M_{0}\right)}
$$

${ }^{1}$ S. Singamaneni, M. C. LeMieux, H. P. Lang, C. Gerber, Y. Lam, S. Zauscher, P. G. Datskos, N. V. Lavrik, H. Jiang, R. R. Naik, T. J. Bunning, and V. V. Tsukruk, Adv. Mater. (Weinheim, Ger.) 20, 653 (2008).

${ }^{2}$ K. M. Goeders, J. S. Colton, and L. A. Bottomley, Chem. Rev. (Washington, D.C.) 108, 522 (2008).

${ }^{3}$ F. P. Beer, E. R. Johnston, and J. T. DeWolf, Mechanics of Materials, 4th ed. (McGraw-Hill, New York, 2006).

${ }^{4}$ R. W. Clough and J. Penzien, Dynamics of Structures, 2nd ed. (McGrawHill, New York, 1993).

${ }^{5}$ J. E. Sader, J. Appl. Phys. 84, 64 (1998).

${ }^{6}$ G. G. Stoney, Proc. R. Soc. London, Ser. A 82, 172 (1909).

${ }^{7}$ S. Timoshenko, J. Opt. Soc. Am. 11, 233 (1925).

${ }^{8}$ R. W. Hoffman, Phys. Thin Films 3, 211 (1966).

${ }^{9}$ P. H. Townsend and D. M. Barnett, J. Appl. Phys. 62, 4438 (1987).

${ }^{10}$ L. B. Freund, J. Cryst. Growth 132, 341 (1993).

${ }^{11}$ L. B. Freund, J. Mech. Phys. Solids 44, 723 (1996).

${ }^{12}$ L. B. Freund, Mater. Res. Soc. Symp. Proc. 436, 393 (1997).

${ }^{13}$ L. B. Freund, J. A. Floro, and E. Chason, Appl. Phys. Lett. 74, 1987 (1999).

${ }^{14}$ Y. Zhang and Y. Zhao, J. Appl. Phys. 99, 053513 (2006).

${ }^{15}$ X. Feng, Y. Huang, and A. J. Rosakis, J. Appl. Mech. 74, 1276 (2007).

${ }^{16}$ Y. Huang and A. J. Rosakis, J. Appl. Mech. 74, 1225 (2007).

${ }^{17}$ X. Feng, Y. Huang, and A. J. Rosakis, J. Appl. Mech. 75, 021022 (2008).

${ }^{18}$ Y. Zhang, J. Appl. Mech. 75, 011008 (2008).

${ }^{19}$ W. Flügge, Viscoelasticity, 2nd ed. (Springer-Verlag, New York, 1975).

${ }^{20}$ A. S. Wineman and K. R. Rajagopal, Mechanical Response of Polymers (Cambridge University Press, Cambridge, 2000).

${ }^{21}$ H. Oberst, Acustica 2, 181 (1952).

${ }^{22}$ F. Schwarzl, Acustica 8, 164 (1958).
${ }^{23}$ U. Sampath, "Analytical modeling of polymer-coated microcantilever dynamic sensors," M.S. thesis, Marquette University, 2005.

${ }^{24}$ R. M. Christensen, Theory of Viscoelasticity, 2nd ed. (Academic, New York, 1982).

${ }^{25}$ M. Fischer, "Analytical modeling of microcantilever-based resonant sensors in lateral flexural mode," M.S. thesis, Marquette University, 2008.

${ }^{26}$ L. R. Senesac, P. Dutta, P. G. Datskos, and M. J. Sepaniak, Anal. Chim. Acta 558, 94 (2006).

${ }^{27}$ A. Loui, T. V. Ratto, T. S. Wilson, S. K. McCall, E. V. Mukerjee, A. H. Love, and B. R. Hart, Analyst (Cambridge, U.K.) 133, 608 (2008).

${ }^{28}$ W. D. Nix, Metall. Trans. A 20A, 2217 (1989).

${ }^{29}$ T. Mura, Micromechanics of Defects in Solids, 2nd ed. (Martinus Nijhoff, Dordrecht, 1987).

${ }^{30}$ M. J. Wenzel, F. Josse, S. M. Heinrich, E. Yaz, and P. G. Datskos, J. Appl. Phys. 103, 064913 (2008).

${ }^{31}$ W. C. Young and R. G. Budynas, Roark's Formulas for Stress and Strain, 7th ed. (McGraw-Hill, New York, 2002).

${ }^{32}$ Z. Hu, T. Thundat, and R. J. Warmack, J. Appl. Phys. 90, 427 (2001).

${ }^{33}$ M. Y. M. Chiang, C. K. Chiang, and W.-L. Wu, J. Eng. Mater. Technol. 124, 274 (2002).

${ }^{34}$ J. E. Sader, J. Appl. Phys. 89, 2911 (2001).

${ }^{35}$ O. Volkersen, Luftfahrtforschung 15, 41 (1938).

${ }^{36}$ E. Suhir, J. Appl. Mech. 56, 595 (1989).

${ }^{37}$ F. Amiot, J. Mech. Mater. Struct. 2, 1787 (2007).

${ }^{38}$ J. Lemaitre, F. A. Leckie, and D. Sherman, Eur. J. Mech. A/Solids 11, 289 (1992).

${ }^{39} \mathrm{P}$. Grütter, personal communication 2008.

${ }^{40}$ M. Godin, O. Laroche, V. Tabard-Cossa, L. Y. Beaulieu, P. Grütter, and P. J. Williams, Rev. Sci. Instrum. 74, 4902 (2003).

${ }^{41}$ M. Godin, P. J. Williams, V. Tabard-Cossa, O. Laroche, L. Y. Beaulieu, R. B. Lennox, and P. Grütter, Langmuir 20, 7090 (2004).

${ }^{42}$ F. Zhou, W. Shu, M. E. Welland, and W. T. S. Huck, J. Am. Chem. Soc. 128, 5326 (2006). 